\title{
Reduction of the Eigenproblem for Hermitian Persymmetric Matrices*
}

\author{
By Marvin J. Goldstein
}

\begin{abstract}
Both computer time and storage can be saved in the calculation of the eigensystem of Hermitian persymmetric matrices if advantage is taken of their special structure.
\end{abstract}

For any vector $v$, let $v^{t}$ denote its transpose and let $J$ denote the permutation matrix obtained by reversing the rows of the identity matrix $I ; J_{i, n+1-i}=I_{i j}$ for $n \times n$ matrices. Note that $J=J^{t}=J^{-1}$.

Definition. $M$ is persymmetric if $J M J=M^{t}$.

Note that all Toeplitz matrices $\left(t_{i j}=t_{i+1, i+1}\right)$ are persymmetric.

Any complex persymmetric matrix $P$ of even order can be written in partitioned form as

$$
P=\left(\begin{array}{ll}
B & C J \\
J F & J B^{t} J
\end{array}\right) \text { with } C=C^{t}, F=F^{t} .
$$

If, in addition, $P$ is Hermitian $\left(P^{t}=\bar{P}\right)$, then we will split it into real and imaginary parts:

$$
P=\left(\begin{array}{cc}
A & H J \\
J H & J A J
\end{array}\right)+i\left(\begin{array}{cc}
S & N J \\
-J N & -J S J
\end{array}\right)
$$

where $A, H, N$ are real symmetric and $S$ is real and skew $\left(S^{t}=-S\right)$. The form of (2) suggests applying $P$ to vectors of special form.

Let $x, y$ be any real vectors conformable with $A$ and write

$$
v=\left(\begin{array}{c}
x \\
J x
\end{array}\right)+i\left(\begin{array}{c}
y \\
-J y
\end{array}\right)
$$

Then, we find that

(4) $\quad P v=\left[\begin{array}{c}(A+H) x+(N-S) y \\ J(A+H) x+J(N-S) y\end{array}\right]+i\left[\begin{array}{r}(N+S) x+(A-H) y \\ -J(N+S) x-J(A-H) y\end{array}\right]$.

Consequently, $v$ will be an eigenvector of $P$ corresponding to an eigenvalue $\lambda$ if and only if

Received February 22, 1973.

AMS (MOS) subject classifications (1970). Primary 15A18, 65F15.

Key words and phrases. Persymmetric, Hermitian, eigenproblem, Toeplitz. meeting.

* This paper is based on a presentation given by the author at the 1972 SIAM-SIGNUM fall 


$$
u \equiv\left(\begin{array}{l}
x \\
y
\end{array}\right)
$$

is an eigenvector corresponding to $\lambda$ (same $\lambda$ ) of the matrix $Q$;

$$
Q \equiv\left(\begin{array}{cc}
A+H & -(S-N) \\
S+N & A-H
\end{array}\right)
$$

Since $S$ is skew, $Q$ is real and symmetric.

Thus, it suffices to solve the eigenproblem for real symmetric $Q$ instead of the complex Hermitian $P$ with obvious savings in both storage and computer time.

The reason that (4) turns out so nicely is that, in (3), we guessed the correct form for an eigenvector. The basic relationship

$$
J P J=P^{t}=\bar{P}
$$

tells us that if $P v=\lambda v$ then

$$
\lambda(J \bar{v})=J \bar{P} \bar{v}=P(J \bar{v})
$$

since $\lambda$ is real.

In particular if $P$ is itself real and symmetric then

$$
N=S=0
$$

and the problem decomposes into two problems of half the original order.

When the order of $P$ is odd the analogous forms are

(7) $P=\left(\begin{array}{ccc}A & c & H J \\ c^{t} & \rho & c^{t} J \\ J H & J c & J A J\end{array}\right)+i\left(\begin{array}{ccc}S & d & N J \\ -d^{t} & 0 & d^{t} J \\ -J N-J d-J S J\end{array}\right), \quad Q=\left(\begin{array}{ccc}A+H \sigma c & -S+N \\ \sigma c^{t} & \rho & \sigma d^{t} \\ S+N \sigma d & A-H\end{array}\right)$

When $P$ is a Toeplitz matrix then so is $A$ but $H$ is a Hankel matrix $\left(h_{i j}=\alpha_{i+i}\right)$.

Acknowledgment. The author is grateful to Professor Beresford N. Parlett for his active interest in this paper and for suggesting the form for this manuscript.

Naval Underwater Systems Center

New London Laboratory

New London, Connecticut 06320 